J. Korean Math. Soc. 49 (2012), No. 6, pp. 1097-1110

http://dx.doi.org/10.4134/JKMS.2012.49.6.1097

\title{
CONVERGENCE PROPERTIES OF THE PARTIAL SUMS FOR SEQUENCES OF END RANDOM VARIABLES
}

\author{
Yongfeng Wu AND Mei GuAN
}

\begin{abstract}
The convergence properties of extended negatively dependent sequences under some conditions of uniform integrability are studied. Some sufficient conditions of the weak law of large numbers, the $p$-mean convergence and the complete convergence for extended negatively dependent sequences are obtained, which extend and enrich the known results in the literature.
\end{abstract}

\section{Introduction and preliminaries}

The concept of negatively dependent (ND) random variables was introduced by Ebrahimi and Ghosh ([4]).

Definition 1.1. The random variables $X_{1}, \ldots, X_{k}$ are said to be negatively upper dependent (NUD) if for all real $x_{1}, \ldots, x_{k}$,

$$
P\left(X_{i}>x_{i}, i=1,2, \ldots, k\right) \leq \prod_{i=1}^{k} P\left(X_{i}>x_{i}\right),
$$

and negatively lower dependent (NLD) if

$$
P\left(X_{i} \leq x_{i}, i=1,2, \ldots, k\right) \leq \prod_{i=1}^{k} P\left(X_{i} \leq x_{i}\right) .
$$

Random variables $X_{1}, \ldots, X_{k}$ are said to be negatively dependent (ND) if they are both NUD and NLD.

Obviously sequences of ND random variables are a family of very wide scope, which contain sequences of independent random variables. Joag-Dev and Proschan ([6]) once pointed out that NA (negatively associated) implies ND, but neither NUD nor NLD implies NA. Since the paper of Joag-Dev and Proschan ([6]) appeared, the convergence properties of ND random sequences

Received April 16, 2010; Revised August 26, 2011.

2010 Mathematics Subject Classification. 60F15.

Key words and phrases. extended negative dependence random sequences, weak law of large numbers, $p$-mean convergence, complete convergence, uniform integrability. 
have been studied by Bozorgnia and Patterson ([2]), Taylor et al. ([13], [14]), Amini and Bozorgnia ([1]), Mi-Hwa Ko et al. ([7], [8]).

Liu ([10]) extended the negatively dependent structure. She introduced the concept of extended negatively dependent (END) random variables.

Definition 1.2. We call random variables $\left\{X_{i}, i \geq 1\right\}$ END if there exists a constant $M>0$ such that both

$$
P\left(X_{i} \leq x_{i}, i=1,2, \ldots, n\right) \leq M \prod_{i=1}^{n} P\left(X_{i} \leq x_{i}\right)
$$

and

$$
P\left(X_{i}>x_{i}, i=1,2, \ldots, n\right) \leq M \prod_{i=1}^{n} P\left(X_{i}>x_{i}\right),
$$

hold for each $n=1,2, \ldots$ and all $x_{1}, \ldots, x_{n}$.

Liu ([10]) pointed out the END structure is substantially more comprehensive than the ND structure in that it can reflect not only a negative dependence structure but also a positive one, to some extent. So it is very significant to study probabilistic properties of this wider END class.

The following examples were provided in Liu ([10]) to illustrate that the extended negative dependence indeed allows a wide range of dependence structures.

Example 1.1. If $\left\{X_{i}, i=1,2\right\}$ and $\left\{X_{i}, i \geq 3\right\}$ are independent of each other, where $X_{1}$ is possibly valued at $x_{1_{1}} \leq x_{1_{2}} \leq \cdots \leq x_{1_{N}}$ and $\left\{X_{i}, i \geq 3\right\}$ is a sequence of mutually independent random variables, then the random variables $\left\{X_{i}, i \geq 1\right\}$ are END. In fact, for any $x_{1}$ and $x_{2}$ such that

$$
P\left(X_{1} \leq x_{1}\right) P\left(X_{2} \leq x_{2}\right)=0 \quad \text { or } \quad P\left(X_{1}>x_{1}\right) P\left(X_{2}>x_{2}\right)=0,
$$

both (1.3) and (1.4) hold trivially. Additionally, for any $x_{1}$ and $x_{2}$ such that

$$
P\left(X_{1} \leq x_{1}\right) P\left(X_{2} \leq x_{2}\right) \neq 0 \quad \text { and } \quad P\left(X_{1}>x_{1}\right) P\left(X_{2}>x_{2}\right) \neq 0,
$$

take

$$
M=1 / \min \left\{P\left(X_{1}=x_{1_{1}}\right), P\left(X_{1}=x_{1_{N}}\right)\right\},
$$

then both (1.3) and (1.4) still hold. Note that there are no dependence restrictions between random variables $X_{1}$ and $X_{2}$.

Example 1.2. For any $n=1,2, \ldots$, let $X_{1}, \ldots, X_{n}$ be dependent according to a copula function $C\left(u_{1}, \ldots, u_{n}\right)$ with absolutely continuous dfs $F_{1}, \ldots, F_{n}$. Assume that the joint copula density

$$
C_{1, \ldots, n}\left(u_{1}, \ldots, u_{n}\right)=\frac{\partial^{n}}{\partial u_{1} \cdots \partial u_{n}} C\left(u_{1}, \ldots, u_{n}\right)
$$


exists and is uniformly bounded in the whole domain. The random variables $\left\{X_{i}, i \geq 1\right\}$ are then END. As noted in Remark 3.1 of Ko and Tang ([9]), for example, copulas in the Frank family of the form

$$
C_{\alpha}\left(u_{1}, \ldots, u_{n}\right)=\frac{1}{\alpha} \ln \left(1+\frac{\left(\mathrm{e}^{\alpha u_{1}}-1\right) \cdots\left(\mathrm{e}^{\alpha u_{n}}-1\right)}{\left(\mathrm{e}^{\alpha}-1\right)^{n-1}}\right), \quad \alpha<0
$$

belong to this category.

Definition 1.3 (Chandra, [9]). Let $\left\{X_{n}, n \geq 1\right\}$ be a sequence of random variables and $p>0$. The sequence $\left\{X_{n}, n \geq 1\right\}$ is said to be uniform integrability in the Cesàro sense if

$$
\lim _{x \rightarrow \infty} \sup _{n \in N} n^{-1} \sum_{k=1}^{n} E\left|X_{k}\right|^{p} I_{\left(\left|X_{k}\right| \geq x\right)}=0 .
$$

Since

$$
\begin{aligned}
E\left|X_{k}\right|^{p} I_{\left(\left|X_{k}\right| \geq x\right)} & =\left(\int_{0}^{x^{p}}+\int_{x^{p}}^{\infty}\right) P\left(\left|X_{k}\right|^{p} I_{\left(\left|X_{k}\right| \geq x\right)}>t\right) \mathrm{d} t \\
& =\int_{0}^{x^{p}} P\left(\left|X_{k}\right| \geq x\right) \mathrm{d} t+\int_{x^{p}}^{\infty} P\left(\left|X_{k}\right|^{p}>t\right) \mathrm{d} t \\
& =x^{p} P\left(\left|X_{k}\right| \geq x\right)+\int_{x^{p}}^{\infty} P\left(\left|X_{k}\right|^{p}>t\right) \mathrm{d} t,
\end{aligned}
$$

we know (1.5) is equivalent to

$$
\lim _{x \rightarrow \infty} \sup _{n \in N} n^{-1} \sum_{k=1}^{n} x^{p} P\left(\left|X_{k}\right| \geq x\right)=0
$$

and

$$
\lim _{x \rightarrow \infty} \sup _{n \in N} n^{-1} \sum_{k=1}^{n} \int_{x^{p}}^{\infty} P\left(\left|X_{k}\right|^{p}>t\right) \mathrm{d} t=0 .
$$

Wu et al. ([15]) studied the weak law of large numbers and the $p$-mean convergence for a sequence of NA random variables under the conditions of (1.5) and (1.6). S. H. Sung et al. ([12]) studied the weak law of large numbers for an array of dependent random variables under some conditions of uniform integrability. The goal of this paper is to study the weak law of large numbers, the $p$-mean convergence and the complete convergence for END sequences under some conditions of uniform integrability in the Cesàro sense. For this goal we need the following lemmas.

Lemma 1.1 (Liu, [10]). If random variables $\left\{X_{i}, i \geq 1\right\}$ are END, then

(1) for any $n=1,2, \ldots$, there exists a constant $M>0$ such that

$$
E\left(\prod_{i=1}^{n} X_{i}^{+}\right) \leq M \prod_{i=1}^{n} E X_{i}^{+}
$$


(2) $\left\{g_{i}\left(X_{i}\right), i=1,2, \ldots\right\}$ are still END, where $\left\{g_{i}(\cdot), i=1,2, \ldots\right\}$ are either all monotone increasing or all monotone decreasing.

Lemma 1.2. Let $\left\{X_{n}, n \geq 1\right\}$ be a sequence of END random variables with mean zero and $0<B_{n}=\sum_{k=1}^{n} E X_{k}^{2}<\infty$. Let $S_{n}=\sum_{k=1}^{n} X_{k}$. Then there exists a constant $M>0$ such that

$$
P\left(\left|S_{n}\right| \geq x\right) \leq \sum_{k=1}^{n} P\left(\left|X_{k}\right| \geq y\right)+2 M \exp \left(\frac{x}{y}-\frac{x}{y} \log \left(1+\frac{x y}{B_{n}}\right)\right)
$$

for $\forall x>0, y>0$.

Proof. The proof is similar to the proof of Theorem 2 in Fuk and Nagaev ([5]). Let $y>0, Y_{i}=\min \left(X_{i}, y\right)$ and $U_{n}=\sum_{i=1}^{n} Y_{i}$. Clearly $E Y_{i} \leq 0, E Y_{i}^{2} \leq E X_{i}^{2}$. By Lemma 1.1(2) for $h>0,\left\{\mathrm{e}^{h Y_{i}}, 1 \leq i \leq n\right\}$ is nonnegative END. Thus, by Lemma 1.1(1), there exists a constant $M>0$ such that

$$
E \mathrm{e}^{h U_{n}}=E \prod_{i=1}^{n} \mathrm{e}^{h Y_{i}} \leq M \prod_{i=1}^{n} E \mathrm{e}^{h Y_{i}} .
$$

Denoting $F_{i}(x)=P\left(X_{i}<x\right)$, then

$$
\begin{aligned}
E \mathrm{e}^{h Y_{i}} & =\int_{-\infty}^{y} \mathrm{e}^{h x} \mathrm{~d} F_{i}(x)+\mathrm{e}^{h y} P\left(X_{i} \geq y\right) \\
& =1+h E Y_{i}+\int_{-\infty}^{y}\left(\mathrm{e}^{h x}-1-h x\right) \mathrm{d} F_{i}(x)+\left(\mathrm{e}^{h y}-1-h y\right) P\left(X_{i} \geq y\right) \\
& \leq 1+\int_{-\infty}^{y}\left(\mathrm{e}^{h x}-1-h x\right) \mathrm{d} F_{i}(x)+\left(\mathrm{e}^{h y}-1-h y\right) P\left(X_{i} \geq y\right)
\end{aligned}
$$

For fixed $h>0$, the function $f(x)=\left(\mathrm{e}^{h x}-1-h x\right) / x^{2}$ is increasing for all $x$. Note that $1+u \leq \mathrm{e}^{u}, \forall u \in R$. Hence

$$
\begin{aligned}
E \mathrm{e}^{h Y_{i}} & \leq 1+\frac{\mathrm{e}^{h y}-1-h y}{y^{2}}\left(\int_{-\infty}^{y} x^{2} \mathrm{~d} F_{i}(x)+y^{2} P\left(X_{i} \geq y\right)\right) \\
& \leq 1+\frac{\mathrm{e}^{h y}-1-h y}{y^{2}} E X_{i}^{2} \leq \exp \left(\frac{\mathrm{e}^{h y}-1-h y}{y^{2}} E X_{i}^{2}\right) .
\end{aligned}
$$

Therefore, by (1.10), for $\forall x>0, \forall h>0$,

$$
\mathrm{e}^{-h x} E \mathrm{e}^{h U_{n}} \leq M \exp \left(-h x+B_{n} \frac{\mathrm{e}^{h y}-1-h y}{y^{2}}\right) .
$$

Letting $h=\log \left(1+\frac{x y}{B_{n}}\right) / y$, we have

$$
\begin{aligned}
\mathrm{e}^{-h x} E \mathrm{e}^{h U_{n}} & \leq M \exp \left(\frac{x}{y}-\frac{x}{y} \log \left(1+\frac{x y}{B_{n}}\right)-\frac{B_{n}}{y^{2}} \log \left(1+\frac{x y}{B_{n}}\right)\right) \\
& \leq M \exp \left(\frac{x}{y}-\frac{x}{y} \log \left(1+\frac{x y}{B_{n}}\right)\right) .
\end{aligned}
$$


Therefore

$$
\begin{aligned}
P\left(S_{n} \geq x\right) & \leq P\left(S_{n} \neq U_{n}\right)+P\left(U_{n} \geq x\right) \\
& \leq \sum_{k=1}^{n} P\left(X_{k} \geq y\right)+\mathrm{e}^{-h x} E \mathrm{e}^{h U_{n}} \\
& \leq \sum_{k=1}^{n} P\left(X_{k} \geq y\right)+M \exp \left(\frac{x}{y}-\frac{x}{y} \log \left(1+\frac{x y}{B_{n}}\right)\right) .
\end{aligned}
$$

Similarly, when $X_{i}$ is replaced by $-X_{i}$, we have

$$
P\left(-S_{n} \geq x\right) \leq \sum_{k=1}^{n} P\left(-X_{k} \geq y\right)+M \exp \left(\frac{x}{y}-\frac{x}{y} \log \left(1+\frac{x y}{B_{n}}\right)\right) .
$$

Therefore, for $\forall x>0, \forall y>0$, we have

$$
\begin{aligned}
P\left(\left|S_{n}\right| \geq x\right) & \leq P\left(S_{n} \geq x\right)+P\left(-S_{n} \geq x\right) \\
& \leq \sum_{k=1}^{n} P\left(\left|X_{k}\right| \geq y\right)+2 M \exp \left(\frac{x}{y}-\frac{x}{y} \log \left(1+\frac{x y}{B_{n}}\right)\right) .
\end{aligned}
$$

The proof is complete.

Lemma 1.3. Let $\left\{X_{n}, n \geq 1\right\}$ be a sequence of random variables satisfying (1.6) for some real number $p>0$. Then

$$
\lim _{n \rightarrow \infty} n^{-\beta / p} \sum_{k=1}^{n} E\left|X_{k}\right|^{\beta} I_{\left(\left|X_{k}\right| \leq n^{1 / p}\right)}=0, \quad \forall \beta>p .
$$

Proof. Put $I=n^{-\beta / p} \sum_{k=1}^{n} E\left|X_{k}\right|^{\beta} I_{\left(\left|X_{k}\right| \leq n^{1 / p}\right)}$. Then

$$
\begin{aligned}
I & =n^{-\beta / p} \sum_{k=1}^{n} \int_{0}^{\infty} P\left(\left|X_{k}\right|^{\beta} I_{\left(\left|X_{k}\right| \leq n^{1 / p}\right)} \geq t\right) \mathrm{d} t \\
& =n^{-\beta / p} \sum_{k=1}^{n} \int_{0}^{n^{\beta / p}} P\left(\left|X_{k}\right|^{\beta} I_{\left(\left|X_{k}\right| \leq n^{1 / p}\right)} \geq t\right) \mathrm{d} t \\
& \leq n^{-\beta / p} \sum_{k=1}^{n} \int_{0}^{n^{\beta / p}} P\left(\left|X_{k}\right|^{\beta} \geq t\right) \mathrm{d} t .
\end{aligned}
$$

Let $t=y^{\beta}$. Then

$$
\begin{aligned}
I & \leq \beta n^{-\beta / p} \sum_{k=1}^{n} \int_{0}^{n^{1 / p}} y^{\beta-1} P\left(\left|X_{k}\right| \geq y\right) \mathrm{d} y \\
& \leq \beta n^{-\beta / p+1} \int_{0}^{n^{1 / p}} y^{\beta-1} n^{-1} \sum_{k=1}^{n} P\left(\left|X_{k}\right| \geq y\right) \mathrm{d} y .
\end{aligned}
$$


By (1.6), for $\forall \varepsilon>0, \exists M>0$ such that when $y>M$, we have

$$
\sup _{n \in N} n^{-1} \sum_{k=1}^{n} P\left(\left|X_{k}\right| \geq y\right) \leq \varepsilon y^{-p} \text {. }
$$

Hence when $n^{1 / p}>M$, we have

$$
\begin{aligned}
I & \leq \beta n^{-\beta / p+1}\left(\int_{0}^{M} y^{\beta-1} n^{-1} \sum_{k=1}^{n} P\left(\left|X_{k}\right| \geq y\right) \mathrm{d} y+\varepsilon \int_{M}^{n^{1 / p}} y^{\beta-p-1} \mathrm{~d} y\right) \\
& \leq \beta n^{-\beta / p+1}\left(C+\frac{\varepsilon}{\beta-p} n^{\beta / p-1}\right)=C \beta n^{-\beta / p+1}+\frac{\beta \varepsilon}{\beta-p} .
\end{aligned}
$$

Since $p<\beta$ and $\varepsilon>0$ is arbitrary, $I \rightarrow 0$ as $n \rightarrow \infty$.

Here in after, the symbol $C$ stands for a generic positive constant which may differ from one place to another. Let $S_{n}=\sum_{k=1}^{n} X_{k}$.

\section{Main results}

Theorem 2.1. Let $1 \leq p<2$ and $\left\{X_{n}, n \geq 1\right\}$ be a sequence of END random variables with $E X_{n}=0$. Then condition (1.6) implies

$$
n^{-1 / p} S_{n} \stackrel{P}{\longrightarrow} 0, \quad n \rightarrow \infty .
$$

Theorem 2.2. Let $1 \leq p<2$ and $\left\{X_{n}, n \geq 1\right\}$ be a sequence of END random variables with $E X_{n}=0$. Then condition (1.5) implies

$$
n^{-1 / p} S_{n} \stackrel{L_{p}}{\longrightarrow} 0, \quad n \rightarrow \infty .
$$

Corollary 2.1. Let $1 \leq p<2$ and $\left\{X_{n}, n \geq 1\right\}$ be a sequence of END random variables with common distribution. Then $E|X|^{p}<\infty$ implies (2.2).

Remark 2.1. Pyke and Root ([11]) obtained the $p$-mean convergence for a sequence of i.i.d. random variables under the same condition of Corollary 2.1. Therefore, Theorem 2.2 extends the result of Pyke and Root ([11]).

Remark 2.2. Wu et al. ([15]) obtained the weak law of large numbers and the $p$-mean convergence for a sequence of NA random variables under the same conditions of Theorems 2.1 and 2.2. Since NA implies ND or ND implies END, Theorems 2.1 and 2.2 extend the results of Wu et al. ([15]).

Theorem 2.3. Let $1 \leq p<2$ and $\left\{X_{n}, n \geq 1\right\}$ be a sequence of END random variables with $E X_{n}=0$. For $\delta>2 / p-1, \alpha p \geq 1$, suppose

$$
\lim _{x \rightarrow \infty} \sup _{n \in N} n^{-1} \sum_{k=1}^{n} x^{1+\delta} P\left(\left|X_{k}\right|^{p} \geq x\right)=0 .
$$

Then

$$
\sum_{n=1}^{\infty} n^{\alpha p-2} P\left(\left|S_{n}\right|>n^{\alpha} \varepsilon\right)<\infty, \quad \forall \varepsilon>0 .
$$


Proof of Theorem 2.1. For any $1 \leq k \leq n$, let

$$
\begin{aligned}
& X_{k}^{\prime}=-n^{1 / p} I_{\left(X_{k} \leq-n^{1 / p}\right)}+X_{k} I_{\left(\left|X_{k}\right|<n^{1 / p}\right)}+n^{1 / p} I_{\left(X_{k} \geq n^{1 / p}\right)}, \\
& X_{k}^{\prime \prime}=X_{k}-X_{k}^{\prime}=\left(X_{k}+n^{1 / p}\right) I_{\left(X_{k} \leq-n^{1 / p}\right)}+\left(X_{k}-n^{1 / p}\right) I_{\left(X_{k} \geq n^{1 / p}\right)}, \\
& S_{n}^{\prime} \widehat{=} \sum_{k=1}^{n} X_{k}^{\prime}, \quad S_{n}^{\prime \prime} \widehat{=} \sum_{k=1}^{n} X_{k}^{\prime \prime} .
\end{aligned}
$$

By Lemma 1.1(2), $X_{k}^{\prime}$ and $X_{k}^{\prime \prime}$ are still END. For $\forall \varepsilon>0$, we have

$$
\begin{aligned}
P\left(n^{-1 / p}\left|S_{n}\right| \geq \varepsilon\right) & \leq P\left(\left|S_{n}^{\prime}-E S_{n}^{\prime}\right| \geq n^{1 / p} \varepsilon / 2\right)+P\left(\left|S_{n}^{\prime \prime}-E S_{n}^{\prime \prime}\right| \geq n^{1 / p} \varepsilon / 2\right) \\
& \widehat{=} I_{1}+I_{2} .
\end{aligned}
$$

Let $B_{n}^{\prime}=\sum_{k=1}^{n} E\left(X_{k}^{\prime}-E X_{k}^{\prime}\right)^{2}$ and $x=y=n^{1 / p} \varepsilon / 2$. By Lemma 1.2 and the Markov inequality, we have

$$
\begin{aligned}
I_{1} & \leq \sum_{k=1}^{n} P\left(\left|X_{k}^{\prime}-E X_{k}^{\prime}\right| \geq n^{1 / p} \varepsilon / 2\right)+\frac{C B_{n}^{\prime}}{B_{n}^{\prime}+n^{2 / p} \varepsilon^{2} / 4} \\
& \leq C n^{-2 / p} B_{n}^{\prime} \leq C n^{-2 / p} \sum_{k=1}^{n} E\left(X_{k}^{\prime}\right)^{2} \\
& \leq C n^{-2 / p} \sum_{k=1}^{n} n^{2 / p} P\left(\left|X_{k}\right| \geq n^{1 / p}\right)+C n^{-2 / p} \sum_{k=1}^{n} E X_{k}^{2} I_{\left(\left|X_{k}\right|<n^{1 / p}\right)} \\
& =C \sum_{k=1}^{n} P\left(\left|X_{k}\right| \geq n^{1 / p}\right)+C n^{-2 / p} \sum_{k=1}^{n} E X_{k}^{2} I_{\left(\left|X_{k}\right|<n^{1 / p}\right)} \\
& \widehat{=} I_{11}+I_{12} .
\end{aligned}
$$

Clearly, (1.6) implies $I_{11} \rightarrow 0$ as $n \rightarrow \infty$. Take $\beta=2$ in (1.11). Then by Lemma $1.3, I_{12} \rightarrow 0$ as $n \rightarrow \infty$. Therefore, $I_{1} \rightarrow 0$ as $n \rightarrow \infty$.

It remains to prove $I_{2} \rightarrow 0$ as $n \rightarrow \infty$. From (1.6) and the definition of $X_{k}^{\prime \prime}$, we have

$$
\begin{aligned}
I_{2} & \leq P\left(\sum_{k=1}^{n}\left|X_{k}^{\prime \prime}-E X_{k}^{\prime \prime}\right| \geq n^{1 / p} \varepsilon / 2\right) \\
& \leq P\left(\exists k ; 1 \leq k \leq n, \text { such that }\left|X_{k}\right| \geq n^{1 / p}\right) \\
& \leq \sum_{k=1}^{n} P\left(\left|X_{k}\right| \geq n^{1 / p}\right) \rightarrow 0 \quad \text { as } n \rightarrow \infty .
\end{aligned}
$$

The proof is complete.

Proof of Theorem 2.2. For $\forall \varepsilon>0$, we have

$$
E\left|n^{-1 / p} S_{n}\right|^{p}=n^{-1} \int_{0}^{\infty} P\left(\left|S_{n}\right|>t^{1 / p}\right) \mathrm{d} t \leq \varepsilon+n^{-1} \int_{\varepsilon n}^{\infty} P\left(\left|S_{n}\right|>t^{1 / p}\right) \mathrm{d} t .
$$


For $t \geq \varepsilon n$, let

$$
\begin{aligned}
& Y_{k}=-t^{1 / p} I_{\left(X_{k} \leq-t^{1 / p}\right)}+X_{k} I_{\left(\left|X_{k}\right|<t^{1 / p}\right)}+t^{1 / p} I_{\left(X_{k} \geq t^{1 / p}\right)}, \\
& Z_{k}=X_{k}-Y_{k}=\left(X_{k}+t^{1 / p}\right) I_{\left(X_{k} \leq-t^{1 / p}\right)}+\left(X_{k}-t^{1 / p}\right) I_{\left(X_{k} \geq t^{1 / p}\right)} .
\end{aligned}
$$

By Lemma 1.1(2), $Y_{k}$ and $Z_{k}$ are still END. Therefore, we have

$$
\begin{aligned}
E\left|n^{-1 / p} S_{n}\right|^{p} \leq & \varepsilon+n^{-1} \int_{\varepsilon n}^{\infty} P\left(\left|\sum_{k=1}^{n} Z_{k}\right|>t^{1 / p} / 2\right) \mathrm{d} t \\
& +n^{-1} \int_{\varepsilon n}^{\infty} P\left(\left|\sum_{k=1}^{n} Y_{k}\right|>t^{1 / p} / 2\right) \mathrm{d} t \\
\widehat{=} & \varepsilon+I_{3}+I_{4} .
\end{aligned}
$$

To prove (2.2), it suffices to prove that $I_{3} \rightarrow 0$ and $I_{4} \rightarrow 0$ as $n \rightarrow \infty$. For $I_{3}$, we can get

$$
\begin{aligned}
I_{3} & \leq n^{-1} \int_{\varepsilon n}^{\infty} P\left(\exists k ; 1 \leq k \leq n, \text { such that }\left|X_{k}\right|>t^{1 / p}\right) \mathrm{d} t \\
& \leq n^{-1} \int_{\varepsilon n}^{\infty} \sum_{k=1}^{n} P\left(\left|X_{k}\right| \geq t^{1 / p}\right) \mathrm{d} t \leq \sum_{k=1}^{n} n^{-1} E\left|X_{k}\right|^{p} I_{\left(\left|X_{k}\right| \geq(\varepsilon n)^{1 / p}\right)} \\
& \leq \sup _{m \in N} m^{-1} \sum_{k=1}^{m} E\left|X_{k}\right|^{p} I_{\left(\left|X_{k}\right| \geq(\varepsilon n)^{1 / p}\right)} \rightarrow 0 \quad \text { as } n \rightarrow \infty .
\end{aligned}
$$

Then we prove $I_{3} \rightarrow 0$ as $n \rightarrow \infty$. Note that $\left|Z_{k}\right| \leq\left|X_{k}\right| I_{\left(\left|X_{k}\right| \geq t^{1 / p}\right)}$. From $E X_{k}=0$ and (1.5), we have

$$
\begin{aligned}
\max _{t \geq \varepsilon n}\left|t^{-1 / p} \sum_{k=1}^{n} E Y_{k}\right| & =\max _{t \geq \varepsilon n}\left|t^{-1 / p} \sum_{k=1}^{n} E Z_{k}\right| \\
& \leq \max _{t \geq \varepsilon n} t^{-1 / p} \sum_{k=1}^{n} E\left|X_{k}\right| I_{\left(\left|X_{k}\right| \geq t^{1 / p}\right)} \\
& \leq(\varepsilon n)^{-1 / p} \sum_{k=1}^{n} E\left|X_{k}\right| I_{\left(\left|X_{k}\right| \geq(\varepsilon n)^{1 / p}\right)} \\
& \leq \varepsilon^{-1} \sup _{m \in N} m^{-1} \sum_{k=1}^{m} E\left|X_{k}\right|^{p} I_{\left(\left|X_{k}\right| \geq(\varepsilon n)^{1 / p}\right)} \rightarrow 0 \text { as } n \rightarrow \infty
\end{aligned}
$$

Therefore, while $n$ is sufficiently large, for $t \geq \varepsilon n$, we have

$$
P\left(\left|\sum_{k=1}^{n} Y_{k}\right|>t^{1 / p} / 2\right) \leq P\left(\left|\sum_{k=1}^{n}\left(Y_{k}-E Y_{k}\right)\right|>t^{1 / p} / 4\right) .
$$


Let $B_{n}^{\prime \prime}=\sum_{k=1}^{n} E\left(Y_{k}-E Y_{k}\right)^{2}, x=t^{1 / p} / 4, y=t^{1 / p} / 4 \gamma, \gamma>p$. By Lemma 1.2 , we have

$$
\begin{aligned}
I_{4} \leq & n^{-1} \int_{\varepsilon n}^{\infty} P\left(\left|\sum_{k=1}^{n}\left(Y_{k}-E Y_{k}\right)\right|>t^{1 / p} / 4\right) \mathrm{d} t \\
\leq & n^{-1} \int_{\varepsilon n}^{\infty} \sum_{k=1}^{n} P\left(\left|Y_{k}-E Y_{k}\right|>t^{1 / p} / 4 \gamma\right) \mathrm{d} t \\
& +C n^{-1} \int_{\varepsilon n}^{\infty}\left(\frac{B_{n}^{\prime \prime}}{B_{n}^{\prime \prime}+t^{2 / p} / 16 \gamma}\right)^{\gamma} \mathrm{d} t \widehat{=} I_{5}+I_{6} .
\end{aligned}
$$

Since

$$
\begin{aligned}
\max _{t \geq \varepsilon n} t^{-1 / p}\left|E Y_{k}\right| & =\max _{t \geq \varepsilon n} t^{-1 / p}\left|E Z_{k}\right| \\
& \leq(\varepsilon n)^{-1 / p} E\left|X_{k}\right| I_{\left(\left|X_{k}\right| \geq(\varepsilon n)^{1 / p}\right)} \\
& \leq(\varepsilon n)^{-1 / p} \sum_{k=1}^{n} E\left|X_{k}\right| I_{\left(\left|X_{k}\right| \geq(\varepsilon n)^{1 / p}\right)} \\
& \leq \varepsilon^{-1} \sup _{m \in N} m^{-1} \sum_{k=1}^{m} E\left|X_{k}\right|^{p} I_{\left(\left|X_{k}\right| \geq(\varepsilon n)^{1 / p}\right)} \rightarrow 0 \text { as } n \rightarrow \infty
\end{aligned}
$$

Hence

$$
\begin{aligned}
I_{5} \leq & n^{-1} \sum_{k=1}^{n} \int_{\varepsilon n}^{\infty} P\left(\left|Y_{k}\right|>t^{1 / p} / 8 \gamma\right) \mathrm{d} t \\
= & n^{-1} \sum_{k=1}^{n} \int_{\varepsilon n}^{\infty} P\left(\left|X_{k}\right| I_{\left(\left|X_{k}\right|<t^{1 / p}\right)}>t^{1 / p} / 8 \gamma\right) \mathrm{d} t \\
& +n^{-1} \sum_{k=1}^{n} \int_{\varepsilon n}^{\infty} P\left(\left|X_{k}\right| \geq t^{1 / p}\right) \mathrm{d} t \\
\widehat{=} & I_{51}+I_{52} .
\end{aligned}
$$

By similar argument as in the proof of $I_{3} \rightarrow 0$, we may prove $I_{52} \rightarrow 0$. For $I_{51}$, we have

$$
\begin{aligned}
I_{51} & =n^{-1} \sum_{k=1}^{n} \int_{\varepsilon n}^{\infty} P\left(\left|X_{k}\right| I_{\left((\varepsilon n)^{1 / p} / 8 \gamma<\left|X_{k}\right|<t^{1 / p}\right)}>t^{1 / p} / 8 \gamma\right) \mathrm{d} t \\
& \leq n^{-1} \sum_{k=1}^{n} \int_{0}^{\infty} P\left(\left|X_{k}\right| I_{\left(\left|X_{k}\right|>(\varepsilon n)^{1 / p} / 8 \gamma\right)}>t^{1 / p} / 8 \gamma\right) \mathrm{d} t \\
& \leq C n^{-1} \sum_{k=1}^{n} E\left|X_{k}\right|^{p} I_{\left(\left|X_{k}\right|>(\varepsilon n)^{1 / p} / 8 \gamma\right)}
\end{aligned}
$$




$$
\leq C \sup _{m \in N} m^{-1} \sum_{k=1}^{m} E\left|X_{k}\right|^{p} I_{\left(\left|X_{k}\right|>(\varepsilon n)^{1 / p} / 8 \gamma\right)} \rightarrow 0 \quad \text { as } n \rightarrow \infty .
$$

Then we prove $I_{6} \rightarrow 0$ as $n \rightarrow \infty$. Clearly, for $x \geq 0, y \geq 0, z \geq 0$ and $\gamma>p \geq 1,(x+y+z)^{\gamma} \leq 3^{\gamma-1}\left(x^{\gamma}+y^{\gamma}+z^{\gamma}\right)$. Hence, by Cr-inequality, we have

$$
\begin{aligned}
I_{6} \leq & C n^{-1} \int_{\varepsilon n}^{\infty}\left(t^{-2 / p} \sum_{k=1}^{n} E X_{k}^{2} I_{\left(\left|X_{k}\right|<t^{1 / p}\right)}+\sum_{k=1}^{n} P\left(\left|X_{k}\right| \geq t^{1 / p}\right)\right)^{\gamma} \mathrm{d} t \\
= & C n^{-1} \int_{\varepsilon n}^{\infty}\left(t^{-2 / p} \sum_{k=1}^{n} E X_{k}^{2} I_{\left(\left|X_{k}\right|<(\varepsilon n)^{1 / p}\right)}\right. \\
& \left.+t^{-2 / p} \sum_{k=1}^{n} E X_{k}^{2} I_{\left.\left((\varepsilon n)^{1 / p}\right) \leq\left|X_{k}\right|<t^{1 / p}\right)}+\sum_{k=1}^{n} P\left(\left|X_{k}\right| \geq t^{1 / p}\right)\right)^{\gamma} \mathrm{d} t \\
\leq & C n^{-1} \int_{\varepsilon n}^{\infty}\left(t^{-2 / p} \sum_{k=1}^{n} E X_{k}^{2} I_{\left(\left|X_{k}\right|<(\varepsilon n)^{1 / p}\right)}\right)^{\gamma} \mathrm{d} t \\
& +C n^{-1} \int_{\varepsilon n}^{\infty}\left(t^{-1 / p} \sum_{k=1}^{n} E\left|X_{k}\right| I_{\left.\left((\varepsilon n)^{1 / p}\right) \leq\left|X_{k}\right|<t^{1 / p}\right)}\right)^{\gamma} \mathrm{d} t \\
& +C n^{-1} \int_{\varepsilon n}^{\infty}\left(\sum_{k=1}^{n} P\left(\left|X_{k}\right| \geq t^{1 / p}\right)\right)^{\gamma} \mathrm{d} t \\
\widehat{=} & I_{61}+I_{62}+I_{63} .
\end{aligned}
$$

Note that (1.5) implies (1.6). Take $\beta=2$ in (1.11), by Lemma 1.3, $p<2$ and $\gamma>p$, we have

$$
\begin{aligned}
I_{61} & =C n^{-1}\left(\sum_{k=1}^{n} E X_{k}^{2} I_{\left(\left|X_{k}\right|<(\varepsilon n)^{1 / p}\right)}\right)^{\gamma} \int_{\varepsilon n}^{\infty} t^{-2 \gamma / p} \mathrm{~d} t \\
& \leq C \varepsilon\left((\varepsilon n)^{-2 / p} \sum_{k=1}^{n} E X_{k}^{2} I_{\left(\left|X_{k}\right|<(\varepsilon n)^{1 / p}\right)}\right)^{\gamma} \rightarrow 0 \text { as } n \rightarrow \infty .
\end{aligned}
$$

By $\gamma>p$, we have

$$
\begin{aligned}
I_{62} & \leq C n^{-1} \int_{\varepsilon n}^{\infty}\left(t^{-1 / p} \sum_{k=1}^{n} E\left|X_{k}\right| I_{\left(\left|X_{k}\right| \geq(\varepsilon n)^{1 / p}\right)}\right)^{\gamma} \mathrm{d} t \\
& \leq C n^{-1}\left(\sum_{k=1}^{n} E\left|X_{k}\right| I_{\left(\left|X_{k}\right| \geq(\varepsilon n)^{1 / p}\right)}\right)^{\gamma} \int_{\varepsilon n}^{\infty} t^{-\gamma / p} \mathrm{~d} t \\
& \leq C \varepsilon\left((\varepsilon n)^{-1 / p} \sum_{k=1}^{n} E\left|X_{k}\right| I_{\left(\left|X_{k}\right| \geq(\varepsilon n)^{1 / p}\right)}\right)^{\gamma} \\
& \leq C \varepsilon\left((\varepsilon n)^{-1} \sum_{k=1}^{n} E\left|X_{k}\right|^{p} I_{\left(\left|X_{k}\right| \geq(\varepsilon n)^{1 / p}\right)}\right)^{\gamma}
\end{aligned}
$$




$$
\leq C \varepsilon^{1-\gamma}\left(\sup _{m \in N} m^{-1} \sum_{k=1}^{m} E\left|X_{k}\right|^{p} I_{\left(\left|X_{k}\right| \geq(\varepsilon n)^{1 / p}\right)}\right)^{\gamma} \rightarrow 0 \quad \text { as } n \rightarrow \infty .
$$

Finally, we prove $I_{63} \rightarrow 0$. Clearly,

$$
\begin{aligned}
\max _{t \geq \varepsilon n} \sum_{k=1}^{n} P\left(\left|X_{k}\right|>t^{1 / p}\right) & \leq \sum_{k=1}^{n} P\left(\left|X_{k}\right|>(\varepsilon n)^{1 / p}\right) \\
& \leq \varepsilon^{-1} n^{-1} \sum_{k=1}^{n} E\left|X_{k}\right|^{p} I_{\left(\left|X_{k}\right| \geq(\varepsilon n)^{1 / p}\right)} \rightarrow 0 \text { as } n \rightarrow \infty .
\end{aligned}
$$

Therefore, while $n$ is sufficiently large, $\sum_{k=1}^{n} P\left(\left|X_{k}\right|>t^{1 / p}\right)<1$ holds uniformly for $t \geq \varepsilon n$. By $\gamma>1$ and similar argument as in the proof of $I_{3} \rightarrow 0$, we can get

$$
\begin{aligned}
I_{63} & \leq C n^{-1} \int_{\varepsilon n}^{\infty} \sum_{k=1}^{n} P\left(\left|X_{k}\right| \geq t^{1 / p}\right) \mathrm{d} t \\
& \leq C \sup _{m \in N} m^{-1} \sum_{k=1}^{m} E\left|X_{k}\right|^{p} I_{\left(\left|X_{k}\right| \geq(\varepsilon n)^{1 / p}\right)} \rightarrow 0 \quad \text { as } n \rightarrow \infty .
\end{aligned}
$$

The proof is complete.

Proof of Theorem 2.3. We follow the notations of $S_{n}^{\prime}$ and $S_{n}^{\prime \prime}$ in the proof of Theorem 2.1. Let

$$
\begin{aligned}
& X_{k}^{\prime}=-x I_{\left(X_{k} \leq-x\right)}+X_{k} I_{\left(\left|X_{k}\right|<x\right)}+x I_{\left(X_{k} \geq x\right)} \\
& X_{k}^{\prime \prime}=X_{k}-X_{k}^{\prime}=\left(X_{k}+x\right) I_{\left(X_{k} \leq-x\right)}+\left(X_{k}-x\right) I_{\left(X_{k} \geq x\right)} .
\end{aligned}
$$

Here we take $x=n^{\alpha(2-p) / 4}$. By Lemma 1.1(2), $X_{k}^{\prime}$ and $X_{k}^{\prime \prime}$ are still END. For any $\varepsilon>0$, we have

$$
\begin{aligned}
& \sum_{n=1}^{\infty} n^{\alpha p-2} P\left(\left|S_{n}\right|>n^{\alpha} \varepsilon\right) \\
\leq & \sum_{n=1}^{\infty} n^{\alpha p-2} P\left(\left|S_{n}^{\prime}-E S_{n}^{\prime}\right|>n^{\alpha} \varepsilon / 2\right)+\sum_{n=1}^{\infty} n^{\alpha p-2} P\left(\left|S_{n}^{\prime \prime}-E S_{n}^{\prime \prime}\right|>n^{\alpha} \varepsilon / 2\right) \\
\widehat{=} & I_{7}+I_{8} .
\end{aligned}
$$

To prove (2.4), it suffices to prove $I_{7}<\infty$ and $I_{8}<\infty$. Note that $\left|X_{k}^{\prime}\right| \leq$ $n^{\alpha(2-p) / 4}$. By similar argument as in the proof of $I_{1} \rightarrow 0$, Lemma 1.2 and the Markov inequality, we have

$$
I_{7} \leq C \sum_{n=1}^{\infty} n^{\alpha p-2-2 \alpha} \sum_{k=1}^{n} E\left(X_{k}^{\prime}\right)^{2} \leq C \sum_{n=1}^{\infty} n^{-1-\alpha(2-p) / 2}<\infty .
$$


Note that $\left|X_{k}^{\prime \prime}\right| \leq\left|X_{k}\right| I_{\left(\left|X_{k}\right| \geq x\right)}$. By Lemma 1.2 and the Markov inequality, we also have

$$
\begin{aligned}
I_{8} \leq & C \sum_{n=1}^{\infty} n^{\alpha p-2-2 \alpha} \sum_{k=1}^{n} E\left|X_{k}\right|^{2} I_{\left(\left|X_{k}\right| \geq x\right)} \\
= & C \sum_{n=1}^{\infty} n^{\alpha p-2-2 \alpha} \sum_{k=1}^{n}\left(\int_{0}^{x^{2}}+\int_{x^{2}}^{\infty}\right) P\left(\left|X_{k}\right|^{2} I_{\left(\left|X_{k}\right| \geq x\right)}>t\right) \mathrm{d} t \\
= & C \sum_{n=1}^{\infty} n^{\alpha p-2-2 \alpha} \sum_{k=1}^{n}\left(\int_{0}^{x^{2}} P\left(\left|X_{k}\right| \geq x\right) \mathrm{d} t+\int_{x^{2}}^{\infty} P\left(\left|X_{k}\right|^{2}>t\right) \mathrm{d} t\right) \\
= & C \sum_{n=1}^{\infty} n^{\alpha p-2-2 \alpha} \sum_{k=1}^{n} x^{2} P\left(\left|X_{k}\right| \geq x\right) \\
& +C \sum_{n=1}^{\infty} n^{\alpha p-2-2 \alpha} \sum_{k=1}^{n} \int_{x^{2}}^{\infty} P\left(\left|X_{k}\right|^{2}>t\right) \mathrm{d} t \\
\widehat{=} & I_{81}+I_{82} .
\end{aligned}
$$

From (2.3), $\exists M>0$, while $x>M$, we have

$$
\sup _{n \in N} n^{-1} \sum_{k=1}^{n} P\left(\left|X_{k}\right|^{p} \geq x\right) \leq x^{-(1+\delta)} .
$$

By $x=n^{\alpha(2-p) / 4},(2.5)$ and $1+\delta-\frac{2}{p}>0$ we have

$$
\begin{aligned}
I_{81} & =\sum_{n=1}^{\infty} n^{\alpha p-2-2 \alpha} \sum_{k=1}^{n} x^{2} P\left(\left|X_{k}\right| \geq x\right) \\
& =\sum_{n=1}^{\infty} n^{\alpha p-2 \alpha-1} n^{-1} \sum_{k=1}^{n} x^{2} P\left(\left|X_{k}\right| \geq x\right) \\
& \leq \sum_{n=1}^{\infty} n^{\alpha p-2 \alpha-1} x^{-p(1+\delta)+2} \\
& =\sum_{n=1}^{\infty} n^{-1-\alpha(2-p)-\alpha p(2-p)\left(1+\delta-\frac{2}{p}\right) / 4}<\infty
\end{aligned}
$$

and

$$
\begin{aligned}
I_{82} & =\sum_{n=1}^{\infty} n^{\alpha p-2 \alpha-1} \int_{x^{2}}^{\infty} n^{-1} \sum_{k=1}^{n} P\left(\left|X_{k}\right|^{2}>t\right) \mathrm{d} t \\
& \leq \sum_{n=1}^{\infty} n^{\alpha p-2 \alpha-1} \int_{x^{2}}^{\infty} t^{-\frac{p}{2}(1+\delta)} \mathrm{d} t \\
& \leq C \sum_{n=1}^{\infty} n^{-1-\alpha(2-p)} x^{-p(1+\delta)+2}
\end{aligned}
$$




$$
\leq C \sum_{n=1}^{\infty} n^{-1-\alpha(2-p)-\alpha p(2-p)\left(1+\delta-\frac{2}{p}\right) / 4}<\infty .
$$

The proof is complete.

Acknowledgments. The authors are grateful to the referee for carefully reading the manuscript and for providing some comments and suggestions which led to improvements in the paper. This work was partially supported by the Anhui Province College Excellent Young Talents Fund Project of China (No. 2011SQRL143) and Humanities and Social Sciences Foundation for The Youth Scholars of Ministry of Education of China (No.12YJCZH217).

\section{References}

[1] D. M. Amini and A. Bozorgnia, Complete convergence for negatively dependent random variables, J. Appl. Math. Stochastic Anal. 16 (2003), no. 2, 121-126.

[2] A. Bozorgnia, R. F. Patterson, and R. L. Taylor, Limit theorems for dependent random variables, World Congress of Nonlinear Analysts '92, Vol. I-IV (Tampa, FL, 1992), 16391650, de Gruyter, Berlin, 1996.

[3] T. K. Chandra, Uniform integrability in the Cesàro sense and the weak law of large numbers, Sankhyā Ser. A 51 (1989), no. 3, 309-317.

[4] N. Ebrahimi and M. Ghosh, Multivariate negative dependence, Comm. Statist. ATheory Methods 10 (1981), no. 4, 307-337.

[5] D. K. Fuk and S. V. Nagaev, Probability inequalities for sums of independent random variables, Theory Probab. Appl. 16 (1971), no. 4, 643-660.

[6] K. Joag-Dev and F. Proschan, Negative association of random variables with applications, Ann. Statist. 11 (1983), no. 1, 286-295.

[7] M. H. Ko, K. H. Han, and T. S. Kim, Strong laws of large numbers for weighted sums of negatively dependent random variables, J. Korean Math. Soc. 43 (2006), no. 6, 13251338.

[8] M. H. Ko and T. S. Kim, Almost sure convergence for weighted sums of negatively orthant dependent random variables, J. Korean Math. Soc. 42 (2005), no. 5, 949-957.

[9] B. Ko and Q. H. Tang, Sums of dependent nonnegative random variables with subexponential tails, J. Appl. Probab. 45 (2008), no. 1, 85-94.

[10] L. Liu, Precise large deviations for dependent random variables with heavy tails, Statist. Probab. Lett. 79 (2009), no. 9, 1290-1298.

[11] R. Pyke and D. Root, On convergence in r-mean of normalized partial sums, Ann. Math. Statist. 32 (1968), no. 9, 379-381.

[12] S. H. Sung, L. Supranee, and A. Volodin, Weak laws of large numbers for arrays under a condition of uniform integrability, J. Korean Math. Soc. 45 (2008), no. 1, 289-300.

[13] R. L. Taylor, R. F. Patterson, and A. Bozorgnia, Weak laws of large numbers for arrays of rowwise negatively dependent random variables, J. Appl. Math. Stochastic Anal. 14 (2001), no. 3, 227-236.

[14] dom variables, Stochastic Anal. Appl. 20 (2002), no. 3, 643-656.

[15] Q. Y. Wu, Y. Q. Wang, and Y. C. Wu, On some limit theorems for sums of NA random matrix sequences, Chin. J. Appl. Probab. Stat. 22 (2006), no. 1, 56-62. 
Yongfeng Wu

Department of Mathematics and Computer Science

TONGLing University

Tongling 244000, P. R. China

E-mail address: wyfwyf@126.com

Mei Guan

Department of Mathematics and Physics

Hefei University

Hefei 230022, P. R. China

E-mail address: guanmei1977@126.com 